\title{
Long-term safety and efficacy of dasatinib in the treatment of chronic-phase chronic myeloid leukemia patients resistant or intolerant to imatinib
}

\author{
This article was published in the following Dove Press journal: \\ Blood and Lymphatic Cancer: Targets and Therapy \\ 18 September 2014 \\ Number of times this article has been viewed
}

\author{
Khalid Shoumariyeh \\ Nikolas von Bubnoff \\ Department of Hematology, Oncology \\ and Stem Cell Transplantation, \\ University Hospital Freiburg, \\ Freiburg, Germany
}

\begin{abstract}
Treatment of chronic myeloid leukemia (CML) has undergone dramatic changes in the last decade. Dissecting the molecular pathways that lead to the development of this disease resulted in the development of targeted therapy against the molecular driver of CML, namely the aberrantly activated tyrosine kinase BCR-ABL1. By introducing the tyrosine kinase inhibitor imatinib to the treatment repertoire, the natural course of the disease has been dramatically altered and overall survival of patients with CML prolonged substantially. Nevertheless, a significant number of patients are primarily resistant, acquire resistance during the course of their disease, or do not tolerate the intake of imatinib due to adverse effects. Second-generation tyrosine kinase inhibitors were developed in an attempt to overcome these problems. Dasatinib is a potent oral kinase inhibitor that was originally developed as an Src-kinase inhibitor but exhibited promising potency against BCR-ABL1 as well. Phase I and II trials demonstrated efficacy in patients failing imatinib, and thus dasatanib was approved in 2006 for the treatment of imatinib-resistant or -intolerant patients with chronic-phase CML harboring the BCR-ABL1 fusion protein. It has since shown promising efficacy and good overall tolerability in subsequent clinical trials, including the Phase III first-line DASISION trial that led to the extension of its approval for first-line treatment of chronic-phase CML. The following review summarizes the available data on the long-term efficacy and safety of dasatinib as a second-line therapy in chronic-phase CML.
\end{abstract}

Keywords: BCR-ABL1, TKI, CML-CP, second-line treatment

\section{Introduction to the management of chronic-phase chronic myeloid leukemia}

Chronic myeloid leukemia (CML) arises by clonal expansion of an aberrant myeloid precursor cell in the bone marrow. It is usually classified into three distinct clinical stages, namely the chronic phase (CP), acceleration phase, and blast crisis, the latter of which resembles acute leukemia and displays a very poor prognosis. ${ }^{1}$ The pathogenetic culprit in CML is the balanced reciprocal translocation between chromosomes 9 and 22 ( $\mathrm{t}[9 ; 22][\mathrm{q} 34 ; 11])$ generating the so-called Philadelphia chromosome, detectable in approximately $95 \%$ of all CML patients. ${ }^{2}$ On a molecular level, the translocation results in the fusion of the c-abl proto-oncogene from chromosome 9 with the breakpoint cluster (BCR)-gene from chromosome 22, yielding an aberrantly activated tyrosine-kinase termed BCR-ABL1. ${ }^{3-5}$ This de novo oncoprotein drives the malignant transformation of the myeloid precursor cell by phosphorylation of downstream effector
Correspondence: Nikolas von Bubnoff Department of Hematology, Oncology and Stem Cell Transplantation, University Hospital Freiburg, Hugstetter Str 55, 79106 Freiburg, Germany

Tel +49 I76 27033210

Email nikolas.bubnoff@uniklinik-freiburg.de 
pathways responsible for growth and increased survival of the precursor cells. ${ }^{6}$

\section{Imatinib in the clinic}

In 2002 treatment of CML-CP underwent a paradigm shift by the introduction and approval of imatinib mesylate (STI571, Gleevec(C) Novartis Pharmaceuticals, Basel, Switzerland), a selective small molecule inhibitor of BCR-ABL1. Additionally, STI571 showed in vitro inhibitory activity against the $\alpha$ - and $\beta$-platelet derived growth factor receptors and c-Kit. ${ }^{7}$ Imatinib specifically binds to the adenosine triphosphate (ATP)-pocket of BCR-ABL1 in its closed conformation, thereby blocking its kinase function. ${ }^{8}$ Several clinical studies demonstrated a durable hematologic and cytogenetic response with a low toxicity profile for imatinib. ${ }^{9,10}$ Follow-up data from the Phase III International Randomized Study of Interferon versus STI571 after 5 and 8 years have confirmed the persistent efficacy of imatinib treatment, with an estimated overall survival (OS) of $85 \%$, while experienced side effects were mostly mild to moderate. ${ }^{11,12}$ However, $15 \%-20 \%$ of Philadelphia chromosome-positive $(\mathrm{Ph}+) \mathrm{CML}-\mathrm{CP}$ patients are resistant to imatinib or develop a resistance in the course of their disease. ${ }^{12}$ Rates of imatinib-resistance rise substantially upon disease progression to about $80 \%$ in patients with blast crisis. ${ }^{13}$ The most common molecular cause of resistance to imatinib is the occurrence of point mutations that yield an open conformation of the tyrosine kinase and thus alter the binding of imatinib to the BCR-ABL oncoprotein. The majority of imatinib-resistance mutations yield amino acid substitutions at one of the following seven residues: M244V, G250E, Y253F/H, E255K/V, T315I, M351T, and F359V, which are located in the ABL tyrosine kinase domain. ${ }^{13}$ Amino acids 248-256 comprise the so-called P-loop, the highly conserved region in the kinase domain that is responsible for binding of ATP. Mutations in one of these residues are found in almost half of the resistant patients harboring a mutation. ${ }^{13}$ In about $10 \%$ of cases, resistance to imatinib occurs due to amplification of the BCR-ABL1 oncogene. ${ }^{14,15}$ BCR-ABL-independent pathways and lack of adherence to the treatment regimens have also been implicated in some cases of resistance. ${ }^{16,17}$ Imatinib resistance is associated with a negative prognostic impact and reduces progression-free survival (PFS) as well as OS of patients with CML. ${ }^{18}$ On the basis of these observations, a search for more potent substances to counteract the permanently activated kinase in CML led to the discovery of new second-generation tyrosine kinase inhibitors (TKI).

\section{Dasatinib in the clinic}

Dasatinib (BMS-354825, SprycelC); Bristol-Myers Squibb, NY, USA) and nilotinib (Tasigna ${ }^{\circledR}$; Novartis Pharmaceuticals, NJ, USA) are two orally available TKI that showed efficacy and were approved for the second-line treatment of CML-CP after imatinib failure due to resistance or intolerance. Dasatinib was originally developed as an Srckinase inhibitor, but displayed potent activity as an ABLinhibitor as well. Moreover, it also binds and suppresses other kinases such as c-Kit or platelet derived growth factor $\beta$-receptor. ${ }^{19}$ As stated above, to exert its inhibitory function, imatinib requires the $\mathrm{ABL}$ tyrosine kinase to be in its closed and thus inactive conformation, whereas dasatinib inhibits the kinase in its active conformation. ${ }^{20}$ In vitro data revealed superior efficacy on wild-type BCR-ABL1 by BMS-354825 compared to imatinib and, intriguingly, also exhibited activity against most of the mutations conferring imatinib-resistance observed in CML patients. The only entirely insensitive BCR-ABL1 mutant found was T315I. ${ }^{21,22}$ Mutants that conferred reduced sensitivity to dasatinib included F317L, V299L, and T315A. ${ }^{23,24}$ Ensuing preclinical and clinical studies demonstrated great potency as dasatinib treatment led to complete hematologic response (CHR) as well as major cytogenetic response (MCyR) in patients with CML-CP resistant or intolerant to imatinib. ${ }^{25}$ Dasatinib showed efficacy in all stages of imatinib-resistant and -intolerant CML. ${ }^{26}$ In 2006 the US Food and Drug Administration approved dasatinib for second-line treatment of patients with CML-CP. Approval of dasatinib was subsequently extended to all phases of CML as well as $\mathrm{Ph}+$ acute lymphoblastic leukemia (ALL) and resistance or intolerance to previous treatment including imatinib, and recently, to newly diagnosed CML-CP. ${ }^{27}$ In this review, we summarize the data on the efficacy and safety of dasatinib as a second-line treatment in CML-CP patients resistant or intolerant to treatment with imatinib.

\section{Efficacy of dasatinib in CML-CP resistant or intolerant to imatinib}

The clinical studies evaluating dasatinib as a second-line treatment in CML-CP after imatinib failure used for this review are summarized in Table 1.

\section{Phase I trial}

The first clinical study to report on dasatinib in a second-line setting in CML-CP was conducted by Talpaz et al. ${ }^{26} \mathrm{~A}$ total of 84 patients with all phases of CML and Ph+ ALL and a history of imatinib intake were selected to receive dasatinib 
Table I Characteristics of clinical trials evaluating efficacy and safety of dasatinib as a second-line treatment in imatinib-resistant or -intolerant CML-CP

\begin{tabular}{|c|c|c|c|c|}
\hline Trial & CAI $80-002^{26,28}$ & START-C ${ }^{29-31}$ & START-R ${ }^{35,36}$ & CAI $80-034^{37-40}$ \\
\hline Study design & Phase I & Phase II & Phase II & Phase III \\
\hline Treatment schedule & I5-I80 mg (QD or BID) & 70 mg (BID) & 70 mg (BID) & $\begin{array}{l}100 \mathrm{mg} \text { QD or } \\
50 \mathrm{mg} \text { BID or } \\
140 \mathrm{mg} \text { QD or } \\
70 \mathrm{mg} \text { BID }\end{array}$ \\
\hline Number of patients (CML-CP) & $n=40$ & $n=387$ & $n=10 \mid$ & $n=662$ \\
\hline Follow-up & 27 months & 24 months & 24 months & 72 months \\
\hline
\end{tabular}

Abbreviations: BID, twice daily; CML-CP, chronic phase-chronic myeloid leukemia; QD, once daily; START-C, SRC/ABL Tyrosine Kinase Inhibition Activity: Research Trials of Dasatinib; START-R, START randomized trial.

in this Phase I dose-escalating study, which focused on the safety and tolerability of dasatinib and gathered evidence for the clinical activity of this second-generation TKI in CML patients after imatinib failure. Of the 84 patients enrolled between November 2003 and April 2005, 40 had CML-CP. An update after a follow-up of at least 27 months was presented as an abstract at the annual meeting of the American Society of Hematology in 2007. ${ }^{28}$ Administered dose of dasatinib ranged between 15 and $180 \mathrm{mg}$ taken daily either as once-daily or twice-daily.

After 27 months of follow-up, 91\% of CML-CP patients achieved a CHR and 51\% a MCyR, with a complete cytogenetic response (CCyR) measured in $44 \%$ (Table 2). There was no statistically significant difference between rates of CCyR for the once-daily and twice-daily schedule arms. In patients who achieved a MCyR during the first year of treatment with dasatinib PFS and OS after 36 months were 87\% and $94 \%$, respectively. PFS and OS declined to $28 \%$ and $68 \%$, respectively, in those patients who achieved no MCyR after 1 year of study treatment (Table 2). ${ }^{28}$ As expected by results from preclinical studies, dasatinib treatment achieved hematologic and cytogenetic responses across all BCR-ABL1 mutations found except for T315I.28

\section{Phase II trials}

The long-term safety profile and efficacy of dasatinib in patients with $\mathrm{Ph}+\mathrm{CML}-\mathrm{CP}$ were investigated on a larger scale by the START (SRC/ABL Tyrosine Kinase Inhibition Activity: Research Trials of Dasatinib) program, which consisted of four single arm international, multicenter, openlabel Phase II clinical studies of dasatinib in all phases of imatinib-resistant or -intolerant $\mathrm{CML}$ and $\mathrm{Ph}+\mathrm{ALL}$ as well as one randomized trial comparing dasatinib with high-dose imatinib in patients with all phases of CML after failure of first-line treatment with imatinib. ${ }^{29-36}$ Initial results from these clinical studies led to dasatinib's approval as a second-line treatment in these entities.

The START in Chronic Phase Patients (START-C) trial assessed efficacy and tolerability of dasatinib in patients with CML-CP with resistance or intolerance to imatinib. In total, 387 patients with CML-CP previously treated with imatinib but intolerant ( $\mathrm{n}=99$ ) or who developed resistance $(n=288)$ were enrolled in this study. Dasatinib was administered at a dose of $140 \mathrm{mg} /$ day ( $70 \mathrm{mg}$ twice-daily). Efficacy was measured by the primary endpoint, the MCyR rate. ${ }^{31}$ The follow-up data after 2 years reported a CHR in 91\% and a MCyR in $62 \%$ of patients, with a CCyR rate of $53 \%$.

Table 2 Long-term efficacy of dasatinib as a second-line treatment in chronic phase CML patients with resistance or intolerance to imatinib

\begin{tabular}{|c|c|c|c|c|}
\hline & CAI $80-002^{26,28}$ & START-C ${ }^{29-31}$ & START-R ${ }^{35,36}$ & CA $180-034^{37-40, a}$ \\
\hline MHR & $91 \%$ & $91 \%$ & $93 \%$ & NA \\
\hline MCyR & $51 \%$ & $62 \%$ & $53 \%$ & $59 \%$ \\
\hline CCyR & $44 \%$ & $53 \%$ & $44 \%$ & $41 \%$ \\
\hline MMR & NA & $47 \%$ & $29 \%$ & $43 \%$ \\
\hline PFS & $87 \%$ b $/ 28 \%^{c}$ & $80 \%$ & $86 \%$ & $49 \%$ \\
\hline OS & $94 \% \mathrm{~b} / 68 \% \mathrm{c}$ & $94 \%$ & NA & $71 \%$ \\
\hline Time point of analysis & $27 / 36$ months & 24 months & 24 months & 72 months \\
\hline
\end{tabular}

Notes: aData shown for patients treated in the $100 \mathrm{mg}$ QD cohort $(\mathrm{n}=167)$; banalysis after 36 months, PFS and OS for patients that achieved a MCyR during the first year of treatment; canalysis after 36 months, PFS and OS for patients that did not achieve a MCyR during the first year of treatment.

Abbreviations: CCyR, complete cytogenetic response; CML, chronic myeloid leukemia; MCyR, major cytogenetic response; MHR, major hematological response; MMR, major molecular response; NA, not available; OS, overall survival; PFS, progression-free survival; QD, once daily; START-C, SRC/ABL Tyrosine Kinase Inhibition Activity: Research Trials of Dasatinib in Chronic Phase Patients; START-R, START randomized trial. 
Major molecular response (MMR), defined by transcript levels of BCR-ABL ${ }^{\text {Is }} \leq 0.10$, was achieved by $47 \%$ of patients (Table 2). In imatinib-resistant patients, MCyR rates were $55 \%$. MCyR was maintained by $88 \%$ of patients at 24 months. Rates of CCyR and MMR in patients intolerant to imatinib were both $78 \%$. Dasatinib-induced responses were durable. At 24 months, PFS in imatinib-resistant and -intolerant patients was $75 \%$ and $94 \%$, respectively. ${ }^{31}$ OS after 24 months was 94\%, with $92 \%$ survivors in the imatinib-resistant group and $100 \%$ in the imatinib-intolerant cohort. Hematological and cytogenetic response was observed across all BCR-ABL genotypes, with the exception of the T315I mutation. ${ }^{31}$

In another international, multicenter, randomized, openlabel, Phase II trial conducted by the START consortium, treatment responses to dasatinib $70 \mathrm{mg}$ twice-daily $(\mathrm{n}=101)$ versus high-dose imatinib ( $800 \mathrm{mg}, \mathrm{n}=49$ ) were investigated in a comparative study in imatinib-resistant CML-CP. ${ }^{35,36}$ The 150 patients randomized (2:1) in the START (START-R) trial had previously been treated with doses of imatinib of 400 to $600 \mathrm{mg}$ daily. Crossover was possible upon progression, lack of MCyR, or intolerance to the treatment to which the patients had been allocated. Randomized patients had been substantially pretreated, having undergone prior therapy with hydroxyurea/anagrelide and/or interferon as well as chemotherapy and even bone marrow transplantation. ${ }^{36}$ Prior to study entry, the majority of patients had been on imatinib for at least $1-3$ years or longer (90\% in both arms). In the dasatinib arm, 51 and 6 patients had a CHR and a MCyR, respectively. In the imatinib arm, 27 patients had a CHR at baseline. After a 2 year follow-up, results clearly favored dasatinib over high-dose imatinib in imatinib-resistant CML-CP. Dasatinib led to higher rates of CHR (93\% versus 82\%), MCyR (53\% versus 33\%) as well as CCyR (44\% versus 18\%). MCyR was maintained in $90 \%$ of the dasatinib-treated and in $74 \%$ of those in the high-dose imatinib cohort after 18 months. ${ }^{36}$ For patients that entered the study without a $\mathrm{CHR}$, the $\mathrm{CHR}$ rate after 24 months was $86 \%$ in the dasatinib arm compared to $72 \%$ in patients treated with $800 \mathrm{mg}$ of imatinib. In those without a MCyR at baseline, MCyR rates were 52\% with dasatinib and 33\% with high-dose imatinib. Of those who achieved a MCyR, $45 \%$ and $18 \%$ of the dasatinib- and imatinib-treated patients, respectively, retained the MCyR after 24 months. ${ }^{36}$ CCyR rates were $42 \%$ and $18 \%$ with dasatinib and imatinib, respectively, in patients not showing a CCyR at baseline. Additionally, those treated with dasatinib achieved a higher MMR than the cohort treated with highdose imatinib (29\% versus $12 \%$ ). Furthermore, in patients presenting a CCyR, the rate of MMR in those treated with dasatinib was $64 \%$ compared to $56 \%$ in the high-dose imatinib group. Crossover from the high-dose imatinib to the dasatinib arm occurred in 39 patients $(80 \%)$, whereas only $20 \%$ of the patients in the dasatinib cohort crossed over to the high-dose imatinib arm. The MCyR rate after crossover was $49 \%$ with dasatinib and $15 \%$ with $800 \mathrm{mg}$ of imatinib. Reasons for discontinuing dasatinib or high-dose imatinib treatment after achieving a MCyR were loss of MCyR in $5 \%$ and $6 \%$, respectively, or treatment intolerance in 3\% of patients receiving dasatinib and $4 \%$ of those receiving imatinib. ${ }^{36}$

\section{Phase III trial}

In the Phase III dose-optimization study, CA180-034, 670 patients with imatinib-resistant $(74 \%)$ or -intolerant (26\%) CML-CP were randomized to either receive dasatinib $100 \mathrm{mg}$ once-daily, $50 \mathrm{mg}$ twice-daily, 140-mg once daily, or $70 \mathrm{mg}$ twice-daily. Of the 670 randomly assigned patients, 662 underwent actual treatment in one of the four dosing arms. Follow-up data from the CA180-034 trial have been published regularly. ${ }^{37-40}$ The most recent analysis was conducted after 72 months, yielding the longest follow-up data on CML-CP patients on dasatinib after imatinib failure. ${ }^{40}$ After 72 months, 28\% ( $\mathrm{n}=188)$ were still on study treatment and eligible for analysis. Of the 188 patients, 144 (77\%) received a once-daily dose. Analysis after 72 months showed that the rates of MCyR ( $59 \%$ versus $55 \%$, respectively) and CCyR ( $41 \%$ versus $45 \%$, respectively) were similar in the $100 \mathrm{mg}$ once-daily and $70 \mathrm{mg}$ twice-daily regimens. ${ }^{40} \mathrm{MMR}$ was achieved by $43 \%$ of patients receiving dasatinib at a dose of $100 \mathrm{mg}$ daily compared to $40 \%$ in all other arms. The estimated PFS and OS rates at the 6 year follow-up were $49 \% / 71 \%, 51 \% / 74 \%, 40 \% / 77 \%$, and $47 \% / 70 \%$ for the $100 \mathrm{mg}$ once-daily, $50 \mathrm{mg}$ twice-daily, $140 \mathrm{mg}$ once-daily, and $70 \mathrm{mg}$ twice-daily treatment schedule, respectively. In patients with $\mathrm{BCR}-\mathrm{ABL}^{\mathrm{IS}} \leq 10 \%$ versus $>10 \%$ at 3 months, rates of PFS and OS were higher after 6 years. The estimated OS rate without transformation to accelerated or blast phase on study treatment was 78\% (76\% with $100 \mathrm{mg}$ once-daily, $80 \%$ with $50 \mathrm{mg}$ twice-daily, $83 \%$ with $140 \mathrm{mg}$ once-daily, and $74 \%$ with $70 \mathrm{mg}$ twice-daily). ${ }^{40}$ During the 6 year follow-up period, ten patients eligible for analysis treated in the $100 \mathrm{mg}$ once-daily study arm demonstrated transformation to accelerated phase or blast phase (nine imatinib-resistant and one imatinib-intolerant). At baseline, 22 patients with more than one dasatinibresistant mutation were revealed by mutational assessment. 
All of those patients were off study treatment at the follow-up after 6 years..$^{40}$

\section{Safety of dasatinib in CML-CP resistant or intolerant to imatinib} Phase I trial

In the Phase I study conducted by Talpaz et al, treatmentrelated hematological toxicity was common in CML-CP patients treated with dasatinib. ${ }^{26} \mathrm{After}$ a median follow-up of 12 months, grade 3 or 4 neutropenia and thrombocytopenia were registered in $45 \%$ and $35 \%$, respectively (Table 3 ). This myelosuppression necessitated treatment interruption in $60 \%$ and dose reduction in $25 \%$ of patients. ${ }^{28}$ The most common grade 3 or 4 nonhematological adverse event during study treatment was pleural effusion, which occurred in 15 patients and required supportive measures including diuretics, thoracocentesis, and pleurodesis. ${ }^{28}$ Remaining adverse events were mostly grade 1 or 2 and were diarrhea, peripheral edema, dyspnea, and headache (Table 4). Grade 3 or 4 liver function abnormalities developed in seven patients but did not require any dose adjustments and resolved by themselves. About $60 \%$ of patients had grade 1 or 2 asymptomatic hypocalcemia. There were no study withdrawals due to adverse events. ${ }^{28}$

\section{Phase II trials}

In line with results from the Phase I study in the START-C trial, grade 3-4 hematologic toxicities were usually observed during the first phases of therapy and included thrombocytopenia (49\%) and neutropenia (50\%). Nonhematological adverse events were generally mild to moderate in severity. Grade 3-4 nonhematological treatment-related side effects primarily consisted of pleural effusion (9\%), dyspnea (6\%), bleeding (4\%), diarrhea (3\%), and fatigue (3\%). ${ }^{31}$

In the START-R trial, grade 3 or 4 adverse events related to the treatment occurred more often in conjunction with dasatinib treatment $(61 \%)$ than high-dose imatinib treatment (39\%). The most frequently observed hematological grade 3 or 4 adverse events were neutropenia, thrombocytopenia, and leucopenia and were more frequent with dasatinib than with high-dose imatinib (Table 3). Nonhematological toxicities were mostly grade 1 or 2 . Grade 3 or 4 nonhematological events consisted of fluid retention, especially pleural effusion, diarrhea, headache, musculoskeletal pain, infection, and fatigue (Table 4). ${ }^{36}$

\section{Phase III trial}

Of all the dosing schedules, $100 \mathrm{mg}$ of dasatinib taken oncedaily displayed the best safety profile in the CA180-034 trial, and thus led to lower rates of treatment discontinuation due to drug toxicity compared to the then standard dose of $70 \mathrm{mg}$ twice-daily ( $11 \%$ versus $21 \%))^{40}$ The most common hematologic adverse events of any grade reported with dasatinib $100 \mathrm{mg}$ once-daily were anemia (89\%), neutropenia (63\%), and thrombocytopenia (60\%). Grade 3-4 hematologic side effects tended to occur early during treatment (in the first year).$^{40}$ Nonhematological adverse events were generally of grade 1 or 2 and usually appeared within the first 2 years of treatment. In line with the Phase I and II studies, the most frequent nonhematological toxicities associated with intake of dasatinib $100 \mathrm{mg}$ once-daily were musculoskeletal pain $(49 \%)$, headache $(47 \%)$, infections $(47 \%)$, diarrhea (41\%), fatigue $(37 \%)$, pleural effusions $(25.3 \%)$, hemorrhage $(24.9 \%)$, and nausea $(22.4 \%)$ (Table 4$).{ }^{40}$ Grade 3 or 4 pleural effusions occurred in $5.3 \%$ of patients treated with $100 \mathrm{mg}$ daily, which was less frequent than in all other schedule arms $(6.6 \%)$. After 72 months, $7 \%$ of patients withdrew from study treatment due to pleural effusions in the $100 \mathrm{mg}$ once-daily arm compared to $9 \%$ in all other arms. ${ }^{40}$ Two patients suffered from pulmonary arterial hypertension, although no diagnostic right-heart catheterization was performed. The results of this trial established $100 \mathrm{mg}$ once-daily as the standard starting dose of dasatinib in CML-CP showing resistance or intolerance to imatinib,

Table 3 Dasatinib-related hematological adverse events in CML-CP after imatinib failure

\begin{tabular}{|c|c|c|c|c|}
\hline & CA $180-002^{26,28}$ & START-C ${ }^{29-31}$ & START-R ${ }^{35,36}$ & CAI $80-034^{37-40}$ \\
\hline Dosing schedule & I5-I80 mg (QD or BID) & $70 \mathrm{mg}$ (BID) & $70 \mathrm{mg}$ (BID) & 100 mg (QD) \\
\hline Grade & Grade 3/4 & Grade 3/4 & Grade 3/4 & Grade 3/4 \\
\hline Anemia & NA & $22 \%$ b & $20 \%$ & $13 \%$ \\
\hline Leukocytopenia & NA & $27 \%^{\mathrm{b}}$ & $24 \%$ & $18 \%$ \\
\hline Neutropenia & $45 \%^{\mathrm{a}}$ & $50 \%{ }^{c}$ & $63 \%$ & $36 \%$ \\
\hline Thrombocytopenia & $35 \%^{a}$ & $49 \%^{c}$ & $57 \%$ & $24 \%$ \\
\hline
\end{tabular}

Notes: a Data after a median follow-up of 12 months; 'bata after a median follow-up of 15.2 months; 'data after a 24-month follow-up.

Abbreviations: BID, twice daily; CML-CP, chronic-phase chronic myeloid leukemia; NA, not available; QD, once daily; START-C, SRC/ABL Tyrosine Kinase Inhibition Activity: Research Trials of Dasatinib in Chronic Phase Patients; START-R, START randomized trial. 
Table 4 Dasatinib-related nonhematological adverse events in CML-CP after imatinib failure

\begin{tabular}{|c|c|c|c|c|c|c|c|c|}
\hline \multirow[b]{2}{*}{ Dosing schedule } & \multicolumn{2}{|c|}{ CAI $80-002^{26,28}$} & \multicolumn{2}{|c|}{ START-C ${ }^{29-32}$} & \multicolumn{2}{|c|}{ START-R $\mathbf{R}^{35,36}$} & \multicolumn{2}{|c|}{ CAI $80-034^{37-40}$} \\
\hline & \multicolumn{2}{|c|}{$\begin{array}{l}\text { I5-I } 80 \text { mg daily } \\
\text { (QD or BID) }\end{array}$} & \multicolumn{2}{|c|}{70 mg daily (BID) } & \multicolumn{2}{|c|}{70 mg daily (BID) } & \multicolumn{2}{|c|}{100 mg daily (QD) } \\
\hline Follow-up & \multicolumn{2}{|l|}{12 months } & \multicolumn{2}{|c|}{ I5.2/24 months } & \multicolumn{2}{|l|}{24 months } & \multicolumn{2}{|l|}{72 months } \\
\hline Grade & All grades & Grade 3/4 & All grades ${ }^{\mathrm{a}}$ & Grade 3/4 & All grades & Grade 3/4 & All grades & Grade $3 / 4$ \\
\hline Pleural effusion & $13 \%$ & 0 & $27 \%$ & $9 \%$ & $25 \%$ & $5 \%$ & $25 \%$ & $5 \%$ \\
\hline Peripheral edema & $18 \%$ & 0 & $18 \%$ & 0 & $20 \%$ & $1 \%$ & $26 \%$ & $1 \%$ \\
\hline Dyspnea & $10 \%$ & 0 & $30 \%$ & $6 \%{ }^{\mathrm{b}}$ & $23 \%$ & $5 \%$ & $33 \%$ & $2 \%$ \\
\hline Abdominal pain & NA & NA & NA & NA & $15 \%$ & 0 & $24 \%$ & $3 \%$ \\
\hline Hemorrhage & NA & NA & NA & $4 \%^{\mathrm{b}}$ & $18 \%$ & $1 \%$ & $25 \%$ & $3 \%$ \\
\hline Fever & NA & NA & $16 \%$ & $1 \%$ & $14 \%$ & 0 & NA & NA \\
\hline Infection & NA & NA & NA & NA & $14 \%$ & $4 \%$ & $47 \%$ & $6 \%$ \\
\hline Diarrhea & $18 \%$ & 0 & $37 \%$ & $3 \%{ }^{\mathrm{b}}$ & $37 \%$ & $3 \%$ & $41 \%$ & $4 \%$ \\
\hline Asthenia & NA & NA & $14 \%$ & $1 \%$ & $15 \%$ & 0 & NA & NA \\
\hline Nausea & $5 \%$ & 0 & $25 \%$ & $1 \%$ & $24 \%$ & 0 & $22 \%$ & $1 \%$ \\
\hline Vomiting & 0 & 0 & $11 \%$ & $0.5 \%$ & $10 \%$ & 0 & NA & NA \\
\hline Fatigue & $8 \%$ & $2 \%$ & $31 \%$ & $3 \%$ & $33 \%$ & $3 \%$ & $37 \%$ & $4 \%$ \\
\hline Headache & $10 \%$ & 0 & $32 \%$ & $1 \%$ & $26 \%$ & $2 \%$ & $47 \%$ & $1 \%$ \\
\hline Skin rash & $2 \%$ & 0 & $26 \%$ & $0.5 \%$ & $18 \%$ & 0 & $33 \%$ & $2 \%$ \\
\hline Musculoskeletal pain & NA & NA & NA & NA & $21 \%$ & $1 \%$ & $49 \%$ & $3 \%$ \\
\hline
\end{tabular}

Notes: "Data for "all grades" after a median follow-up of 15.2 months; bdata from a 24-month follow-up presented as an abstract at the 2008 American Society of Clinical Oncology Annual Meeting."

Abbreviations: BID, twice daily; CML-CP, chronic-phase chronic myeloid leukemia; NA, not available; QD, once daily; START-C, SRC/ABL Tyrosine Kinase Inhibition Activity: Research Trials of Dasatinib in Chronic Phase Patients; START-R, START randomized trial.

since that dose was associated with fewer treatment-related toxicities, while showcasing efficacy comparable to that of $70 \mathrm{mg}$ twice-daily.

\section{Conclusion}

CML constitutes the first disease in which better knowledge of the underlying molecular pathogenesis led to the development of a specific targeted therapy in the form of imatinib. Longterm follow-up data from clinical studies testing imatinib in CML-CP identified a group of patients with CML that does not profit sufficiently from this tailored inhibitor due to primary or secondary resistance to imatinib or intolerance because of adverse effects. ${ }^{41,42}$ Different strategies have been deployed to overcome these obstacles in TKI-based CML treatment. The available therapeutic options in the resistant setting were limited until new and more potent TKI were developed, such as dasatinib, nilotinib, and recently bosutinib and ponatinib, with ponatinib currently the only TKI with T315I activity. ${ }^{43}$ Dasatinib displays more potent suppressive activity against wild-type BCR-ABL than imatinib and was approved for the second-line treatment of CML-CP in patients intolerant or resistant to imatinib. The long-term data presented in this review confirm the notion that dasatinib exhibits durable efficacy in CML-CP after imatinib failure, while maintaining a relatively safe toxicity profile, with adverse events being mostly of grade 1 or 2 . Consistent with preclinical data from in vitro investigations, dasatinib demonstrated efficacy in most genetic mutations conferring imatinib-resistance, with the exception of T315I. ${ }^{44}$

Noteworthy, sequential treatment with dasatinib in a second-line setting after resistance-induced failure of imatinib is associated with the emergence of drug-specific BCRABL kinase domain mutations not detectable prior to the initiation of dasatinib such as V299L, T315A, F317L/V/I/C, and compound mutations. ${ }^{45,46}$ These mutations are associated with a poor outcome of dasatinib treatment while acquired mutations in $\mathrm{Y} 253 \mathrm{H}, \mathrm{E} 255 \mathrm{~K} / \mathrm{V}, \mathrm{F} 359 \mathrm{~V} / \mathrm{C} / \mathrm{I}$, or T315I are frequently detected in patients with failure during nilotinib treatment. Intriguingly, the presence of certain mutations is not always a good predictor of treatment outcome, as shown by a case report by Oyekunle et al ${ }^{47}$ In their presented case, a F317L mutation was first detected while a CML-CP patient was undergoing treatment with dasatinib. Treatment with dasatinib was continued, and a sustained MMR was achieved while the F317L mutation was not detectable in subsequent kinase domain mutation analyses, suggesting other factors involved in determining treatment outcome. Nonetheless, recent European Leukemia Network guidelines recommend a mutational analysis for possible BCR-ABL1 kinase domain point mutations if hematologic or cytogenetic failure occurs during TKI treatment to identify the most suitable TKI based on the identified mutations. ${ }^{48,49}$ 
Dasatinib led to a rapid and deep hematological, cytological, and molecular response in both first- and second-line treatment of CML-CP. ${ }^{36,50}$ Response rates at 3 and 6 months after treatment initiation are being increasingly recognized as predictive markers for low transformation rates into accelerated or blast phases, translating into better longterm outcome and survival. ${ }^{40,51-53}$ Moreover, an early switch to dasatinib after imatinib failure seems advisable to yield an optimal response and facilitate best long-term outcome, as shown by Quintás-Cardama et al in their retrospective pooled analysis of the START-C, START-R, and CA180-034 trials. ${ }^{54}$ Their analysis revealed that the best outcome after progression on imatinib treatment was achieved if dasatinib was administered early after MCyR loss instead of switching to dasatinib after the loss of both MCyR and CHR.

In the Phase III dose-optimizing study by Shah et al, $100 \mathrm{mg}$ of dasatinib taken once-daily showcased the best safety profile, while achieving the same efficacy as the other dosing schedules in imatinib-resistant or -intolerant patients with CML-CP. ${ }^{40}$

Treatment with dasatinib was generally well tolerated. Myelosuppression was frequently reported with dasatinib but was usually reversible and manageable by dose modifications. Nonhematological grade 1 or 2 adverse events such as musculoskeletal pain, headache, diarrhea, peripheral edema, fatigue, and nausea were commonly observed across all studies. The most common nonhematological grade 3 or 4 side effects from dasatinib treatment were pleural effusions..$^{55-57}$ As shown by the Phase III dose finding trial by Shah et al, the incidence of pleural effusions was lower in patients receiving a dose of $100 \mathrm{mg}$ once-daily than in those given $70 \mathrm{mg}$ twice-daily dosing regimen. ${ }^{40}$

Remarkably, treatment discontinuation occurred at rather high rates across all studies. This owes at least partially to the fact that the patient collective assessed in these studies had usually been heavily pretreated before therapy with dasatinib was initiated.

In summary, dasatinib treatment yields durable, longterm, major hematological, cytogenetic as well as molecular responses as a second-line treatment in CML-CP patients resistant or intolerant to imatinib. The long-term use of dasatinib is generally well tolerated. It is efficacious against most known BCR-ABL1 mutations conferring resistance to imatinib. In addition to its use in newly diagnosed CML-CP, dasatinib provides a safe and highly effective treatment option after initial failure of imatinib, and thus represents a valuable addition to the armamentarium of available first- and secondline BCR-ABL1 inhibitors.

\section{Disclosure}

The authors report no conflicts of interest in this work.

\section{References}

1. Faderl S, Talpaz M, Estrov Z, Kantarjian HM. Chronic myelogenous leukemia: biology and therapy. Ann Intern Med. 1999;131(3):207-219.

2. Rowley JD. Letter. A new consistent chromosomal abnormality in chronic myelogenous leukaemia identified by quinacrine fluorescence and Giemsa staining. Nature. 1973;243(5405):290-293.

3. Bartram CR, de Klein A, Hagemeijer A, et al. Translocation of c-ab1 oncogene correlates with the presence of a Philadelphia chromosome in chronic myelocytic leukaemia. Nature. 1983;306(5940):277-280.

4. Konopka JB, Watanabe SM, Witte ON. An alteration of the human c-abl protein in K562 leukemia cells unmasks associated tyrosine kinase activity. Cell. 1984;37(3):1035-1042.

5. Lugo TG, Pendergast AM, Muller AJ, Witte ON. Tyrosine kinase activity and transformation potency of bcr-abl oncogene products. Science. 1990;247(4946):1079-1082.

6. Melo JV, Deininger MW. Biology of chronic myelogenous leukemia - signaling pathways of initiation and transformation. Hematol Oncol Clin North Am. 2004;18(3):545-568, vii.

7. Buchdunger E, Cioffi CL, Law N, et al. Abl protein-tyrosine kinase inhibitor STI571 inhibits in vitro signal transduction mediated by c-kit and platelet-derived growth factor receptors. J Pharmacol Exp Ther. 2000;295(1):139-145.

8. Schindler T, Bornmann W, Pellicena P, Miller WT, Clarkson B, Kuriyan J. Structural mechanism for STI-571 inhibition of abelson tyrosine kinase. Science. 2000;289(5486):1938-1942.

9. O'Brien SG, Deininger MW. Imatinib in patients with newly diagnosed chronic-phase chronic myeloid leukemia. Semin Hematol. 2003; 40(2 Suppl 2):26-30.

10. Kantarjian HM, Cortes JE, O’Brien S, et al. Long-term survival benefit and improved complete cytogenetic and molecular response rates with imatinib mesylate in Philadelphia chromosome-positive chronic-phase chronic myeloid leukemia after failure of interferon-alpha. Blood. 2004;104(7):1979-1988.

11. Druker BJ, Guilhot F, O’Brien SG, et al; IRIS Investigators. Five-year follow-up of patients receiving imatinib for chronic myeloid leukemia. N Engl J Med. 2006;355(23):2408-2417.

12. Deininger $\mathrm{M}, \mathrm{O}$ 'Brien $\mathrm{SG}$, Guilhot $\mathrm{F}$, et al. International randomize study of interferon vs STI571 (IRIS) 8-year follow-up: sustained survival and low risk for progression or events in patients with newly diagnosed chronic myeloid leukemia in chronic phase (CML-CP) treated with imatinib. Blood. (ASH Annual Meeting Abstracts) 2009;114(22): Abstract 1126

13. Soverini S, Colarossi S, Gnani A, et al. Contribution of ABL kinase domain mutations to imatinib resistance in different subsets of Philadelphiapositive patients: by the GIMEMA Working Party on chronic myeloid leukemia. Clin Cancer Res. 2006;12(24):7374-7379.

14. Gorre ME, Mohammed M, Ellwood K, et al. Clinical resistance to STI-571 cancer therapy caused by BCR-ABL gene mutation or amplification. Science. 2001;293(5531):876-880.

15. Weisberg E, Griffin JD. Mechanism of resistance to the ABL tyrosine kinase inhibitor STI571 in BCR/ABL-transformed hematopoietic cell lines. Blood. 2000;95(11):3498-3505.

16. Donato NJ, Wu JY, Stapley J, et al. BCR-ABL independence and LYN kinase overexpression in chronic myelogenous leukemia cells selected for resistance to STI571. Blood. 2003;101(2):690-698.

17. Marin D, Bazeos A, Mahon FX, et al. Adherence is the critical factor for achieving molecular responses in patients with chronic myeloid leukemia who achieve complete cytogenetic responses on imatinib. J Clin Oncol. 2010;28(14):2381-2388.

18. Kantarjian H, O'Brien S, Talpaz M, et al. Outcome of patients with Philadelphia chromosome-positive chronic myelogenous leukemia post-imatinib mesylate failure. Cancer. 2007;109(8):1556-1560. 
19. Lombardo LJ, Lee FY, Chen P, et al. Discovery of N-(2-chloro6-methyl- phenyl)-2-(6-(4-(2-hydroxyethyl)- piperazin-1-yl)-2methylpyrimidin-4- ylamino)thiazole-5-carboxamide (BMS-354825), a dual Src/Abl kinase inhibitor with potent antitumor activity in preclinical assays. J Med Chem. 2004;47(27):6658-6661.

20. Vajpai N, Strauss A, Fendrich G, et al. Solution conformations and dynamics of ABL kinase-inhibitor complexes determined by NMR substantiate the different binding modes of imatinib/nilotinib and dasatinib. J Biol Chem. 2008;283(26):18292-18302.

21. Shah NP, Tran C, Lee FY, Chen P, Norris D, Sawyers CL. Overriding imatinib resistance with a novel ABL kinase inhibitor. Science. 2004;305(5682):399-401.

22. O'Hare T, Walters DK, Stoffregen EP, et al. In vitro activity of BcrAbl inhibitors AMN107 and BMS-354825 against clinically relevant imatinib-resistant Abl kinase domain mutants. Cancer Res. 2005;65(11): $4500-4505$.

23. O'Hare T, Eide CA, Deininger MW. Bcr-Abl kinase domain mutations, drug resistance, and the road to a cure for chronic myeloid leukemia. Blood. 2007;110(7):2242-2249.

24. Müller MC, Cortes JE, Kim DW, et al. Dasatinib treatment of chronicphase chronic myeloid leukemia: analysis of responses according to preexisting BCR-ABL mutations. Blood. 2009;114(24):4944-4953.

25. Hochhaus A, Kantarjian HM, Baccarani M, et al. Dasatinib induces notable hematologic and cytogenetic responses in chronic-phase chronic myeloid leukemia after failure of imatinib therapy. Blood. 2007;109(6): 2303-2309.

26. Talpaz M, Shah NP, Kantarjian H, et al. Dasatinib in imatinibresistant Philadelphia chromosome-positive leukemias. $N$ Engl J Med. 2006;354(24):2531-2541

27. Amrein PC. First-line therapy of chronic myeloid leukemia - focus on dasatinib. Blood and Lymphatic Cancer: Targets and Therapy. 2012;2: $77-85$.

28. Cortes J, Sawyers CL, Kantarjian HM, et al. Long-term efficacy of dasatinib in chronic-phase CML: results from the Phase I trial (CA180002). Blood. (ASH Annual Meeting Abstracts) 2007;110(11): Abstract 1026.

29. Hochhaus A, Baccarani M, Deininger M, et al. Dasatinib induces durable cytogenetic responses in patients with chronic myelogenous leukemia in chronic phase with resistance or intolerance to imatinib. Leukemia. 2008;22(6):1200-1206.

30. Hochhaus A, Müller MC, Radich J, et al. Dasatinib-associated major molecular responses in patients with chronic myeloid leukemia in chronic phase following imatinib failure: response dynamics and predictive value. Leukemia. 2009;23(9):1628-1633.

31. Mauro MJ, Baccarani M, Cervantes F, et al. Dasatinib 2-year efficacy in patients with chronic-phase chronic myelogenous leukemia (CML-CP) with resistance or intolerance to imatinib (START-C). J Clin Oncol. 2008;26(Suppl 15): Abstract 7009.

32. Apperley JF, Cortes JE, Kim DW, et al. Dasatinib in the treatment of chronic myeloid leukemia in accelerated phase after imatinib failure: the START a trial. J Clin Oncol. 2009;27(21):3472-3479.

33. Saglio G, Dombret H, Rea D, et al. Dasatinib efficacy in patients with imatinib-resistant/-intolerant chronic myeloid leukemia in blast phase: 24-month data from the START program. Haematologica. 2008;93(Suppl 1):349. Abstract 0880.

34. Ottmann O, Dombret H, Martinelli G, et al. Dasatinib induces rapid hematologic and cytogenetic responses in adult patients with Philadelphia chromosome positive acute lymphoblastic leukemia with resistance or intolerance to imatinib: interim results of a phase 2 study. Blood. 2007;110(7):2309-2315.

35. Kantarjian H, Pasquini R, Hamerschlak N, et al. Dasatinib or high-dose imatinib for chronic-phase chronic myeloid leukemia after failure of first-line imatinib: a randomized phase 2 trial. Blood. 2007;109(12): $5143-5150$

36. Kantarjian HM, Pasquini R, Lévy V, et al. Dasatinib or high-dose imatinib for chronic-phase chronic myeloid leukemia resistant to imatinib at a dose of 400 to 600 milligrams daily: two-year follow-up of a randomized phase 2 study (START-R). Cancer. 2009;115(18):4136-4147.
37. Shah NP, Kim DW, Kantarjian H, et al. Potent, transient inhibition of $\mathrm{BCR}-\mathrm{ABL}$ with dasatinib $100 \mathrm{mg}$ daily achieves rapid and durable cytogenetic responses and high transformation-free survival rates in chronic phase chronic myeloid leukemia patients with resistance, suboptimal response or intolerance to imatinib. Haematologica. 2010;95(2):232-240.

38. Stone RM, Kim DW, Kantarjian HM, et al. Dasatinib dose-optimization study in chronic phase chronic myeloid leukemia (CML-CP): three-year follow-up with dasatinib $100 \mathrm{mg}$ once daily and landmark analysis of cytogenetic response and progression-free survival (PFS). J Clin Oncol. 2009;27(Suppl 15): Abstract 7007.

39. Shah NP, Cortes JE, Schiffer CA, et al. Five-year follow-up of patients with imatinib-resistant or intolerant chronic-phase chronic myeloid leukemia (CML-CP) receiving dasatinib. J Clin Oncol. 2011;29(Suppl 15): Abstract 6512.

40. Shah NP, Guilhot F, Cortes JE, et al. Long-term outcome with dasatinib after imatinib failure in chronic-phase chronic myeloid leukemia: follow-up of a phase 3 study. Blood. 2014;123(15):2317-2324.

41. Lucas CM, Wang L, Austin GM, et al. A population study of imatinib in chronic myeloid leukaemia demonstrates lower efficacy than in clinical trials. Leukemia. 2008;22(10):1963-1966.

42. de Lavallade H, Apperley JF, Khorashad JS, et al. Imatinib for newly diagnosed patients with chronic myeloid leukemia: incidence of sustained responses in an intention-to-treat analysis. $J$ Clin Oncol. 2008;26(20):3358-3363.

43. Cortes JE, Kantarjian H, Shah NP, et al. Ponatinib in refractory Philadelphia chromosome-positive leukemias. $N$ Engl J Med. 2012;367(22):2075-2088

44. Jabbour E, Jones D, Kantarjian HM, et al. Long-term outcome of patients with chronic myeloid leukemia treated with second-generation tyrosine kinase inhibitors after imatinib failure is predicted by the in vitro sensitivity of BCR-ABL kinase domain mutations. Blood. 2009;114(10): 2037-2043.

45. Soverini S, Gnani A, Colarossi S, et al. Philadelphia-positive patients who already harbor imatinib-resistant Bcr-Abl kinase domain mutations have a higher likelihood of developing additional mutations associated with resistance to second- or third-line tyrosine kinase inhibitors. Blood. 2009;114(10):2168-2171

46. Bauer RC, Sänger J, Peschel C, DuysterJ, von Bubnoff N. Sequential inhibitor therapy in CML: in vitro simulation elucidates the pattern of resistance mutations after second- and third-line treatment. Clin Cancer Res. 2013;19(11):2962-2972.

47. Oyekunle AA, Castagnetti F, Gugliotta G, Soverini S, Baccarani M, Rosti G. F317L BCR-ABL1 kinase domain mutation associated with a sustained major molecular response in CML patient on dasatinib. Leuk Res. 2011;35(7):e118-e120.

48. Soverini S, Hochhaus A, Nicolini FE, et al. BCR-ABL kinase domain mutation analysis in chronic myeloid leukemia patients treated with tyrosine kinase inhibitors: recommendations from an expert panel on behalf of the European LeukemiaNet. Blood. 2011;118(5):1208-1215.

49. Baccarani M, Deininger MW, Rosti G, et al. European LeukemiaNet recommendations for the management of chronic myeloid leukemia: 2013. Blood. 2013;122(6):872-884

50. Jabbour E, Kantarjian HM, Saglio G, et al. Early response with dasatinib or imatinib in chronic myeloid leukemia: 3-year follow-up from a randomized phase 3 trial (DASISION). Blood. 2013;123(4):494-500.

51. Milojkovic D, Apperley JF, Gerrard G, et al. Responses to second-line tyrosine kinase inhibitors are durable: an intention-to-treat analysis in chronic myeloid leukemia patients. Blood. 2012;119(8):1838-1843.

52. Quintás-Cardama A, Kantarjian H, Jones D, et al. Delayed achievement of cytogenetic and molecular response is associated with increased risk of progression among patients with chronic myeloid leukemia in early chronic phase receiving high-dose or standard-dose imatinib therapy. Blood. 2009;113(25):6315-6321.

53. Jabbour E, Kantarjian H, O'Brien S, et al. The achievement of an early complete cytogenetic response is a major determinant for outcome in patients with early chronic phase chronic myeloid leukemia treated with tyrosine kinase inhibitors. Blood. 2011;118(17):4541-4546; quiz 4759. 
54. Quintás-Cardama A, Cortes JE, O’Brien S, et al. Dasatinib early intervention after cytogenetic or hematologic resistance to imatinib in patients with chronic myeloid leukemia. Cancer. 2009;115(13): 2912-2921.

55. Brave M, Goodman V, Kaminskas E, et al. Sprycel for chronic myeloid leukemia and Philadelphia chromosome-positive acute lymphoblastic leukemia resistant to or intolerant of imatinib mesylate. Clin Cancer Res. 2008;14(2):352-359.
56. Porkka K, Khoury HJ, Paquette RL, Matloub Y, Sinha R, Cortes JE. Dasatinib $100 \mathrm{mg}$ once daily minimizes the occurrence of pleural effusion in patients with chronic myeloid leukemia in chronic phase and efficacy is unaffected in patients who develop pleural effusion. Cancer. 2010;116(2):377-386.

57. Kim D, Goh HG, Kim SH, Cho BS, Kim DW. Long-term pattern of pleural effusion from chronic myeloid leukemia patients in second-line dasatinib therapy. Int J Hematol. 2011;94(4):361-371.

\section{Publish your work in this journal}

Blood and Lymphatic Cancer: Targets and Therapy is an international, peer-reviewed, open access journal focusing on blood and lymphatic cancer research, identification of therapeutic targets and the optima use of preventative and integrated treatment interventions to achieve improved outcomes, enhanced survival and quality of life for the cancer patient. The manuscript management system is completely online and includes a very quick and fair peer-review system. Visit http://www.dovepress.com/testimonials.php to read real quotes from published authors.

Submit your manuscript here: http://www.dovepress.com/blood-and-lymphatic-cancer-targets-and-therapy-journal 